\title{
Surveys for active galaxies: discovery and studies
}

\author{
A. M. Mickaelian* H. V. Abrahamyan, G. M. Paronyan, G. A. Mikayelyan, and M. V. \\ Gyulzadyan
}

NAS RA Byurakan Astrophysical Observatory (BAO), Byurakan 0213, Aragatzotn Province, Armenia

\begin{abstract}
We present surveys and related studies of active galaxies carried out at the Byurakan Astrophysical Observatory (BAO). This was one of the main research subjects at BAO during many years, since mid-1950s, when Viktor Ambartsumian suggested the hypothesis of the activity of the galactic nuclei. A number of surveys and searches for Active Galactic Nuclei (AGN) and other active galaxies were accomplished during 1960s-1980s. Since mid-1990s, our research group carried out new surveys and studies of active galaxies based on the First Byurakan Survey (FBS or Markarian Survey) and then a number of others. Here we also present the recent results of studies on active galaxies (both AGN and Starbursts) by the Extragalactic group of the Byurakan Astrophysical Observatory (BAO) Research Department "Astronomical Surveys". These studies are characterized by multiwavelength approach to statistical analysis of large amount of data obtained in different wavelengths; from X-ray to radio. A fine classification scheme for active galaxies has also been suggested.
\end{abstract}

Keywords: active galaxies, AGN, Starburst Galaxies, quasars, Seyfert galaxies, LINERs, composite spectrum objects, HII, IRAS galaxies, variable sources

\section{Introduction}

The Byurakan Astrophysical Observatory has always been famous for surveys for active galaxies. Such search works started just after Viktor Ambartsumian's hypothesis on Active Galactic Nuclei (AGN) in mid-1950s. Here we give a list of the most important surveys carried out in 1960s-2000s.

\section{Byurakan Surveys for Active Galaxies}

- First Byurakan Survey (FBS) - Markarian Survey, 1515 UVX galaxies B. E. Markarian, V. A. Lipovetsky, J. A. Stepanian, 1965-1980: 1515 UV-excess (UVX) galaxies, Markarian galaxies (Markarian et al., 1989, Mazzarella \& Balzano, 1986)

- High surface brightness galaxies, 621 Arakelian galaxies; Arakelian (1975)

- Second Byurakan Survey (SBS)

B. E. Markarian, J. A. Stepanian, V. A. Lipovetsky, L. K. Erastova, V. H. Chavushian, 197891: UVX and emission line gals., QSOs ( 23600 objects, incl. 1800 gals; 600 QSOs, 170 Sy, 12 BLL); Markarian et al. (1987)

- Shahbazian's compact groups of compact galaxies; Baier et al. (1974)

- Kazarian survey: 706 UVX (Kazarian) galaxies; Kazarian et al. (2010)

- Second Part of the FBS: Blue Stellar Objects (FBS BSOs), 1103 objects

H. V. Abrahamian, A. M. Mickaelian, 1987-1996: QSOs and Seyferts

*aregmick@yahoo.com 
- Optical identification and study of IRAS sources, 1278 BIG objects A. M. Mickaelian et al., 1995-2004: ULIRGs, AGN and SB

- Optical identification and study of ROSAT sources; 4253 ROSAT AGN A. M. Mickaelian et al., 2002-2006: AGN and SB

Recent results of the Extragalactic group of BAO Research Department "Astronomical Surveys" are related to multiwavelength studies of active galaxies using large amount of data from X-ray, UV, optical, IR and radio ranges, with heavy use of cross-correlations, classifications on activity types using our observations and SDSS spectra (Ahumada et al., 2020), building diagnostic diagrams, Spectral Energy Distributions (SEDs), etc. Results on HRC/BHRC sample objects (optical identifications of ROSAT X-ray sources), studies of Markarian galaxies in UV and multiwavelength SEDs, abundance and star formation determinations in Mrk galaxies from SDSS spectra, revised optical classification of "LINERs", study and classification of SDSS spectra for Byurakan-IRAS Galaxies (BIG objects), summary of observations and study of Byurakan-IRAS Galaxies, discovery of new bright ULIRGs from the IRAS PSC/FSC Combined Catalogue (Abrahamyan et al., 2015) and their spectral classification using SDSS spectra, radio variable sources at $1400 \mathrm{MHz}$ and their optical variability, classification of BZCAT objects having uncertain types (BZU objects), and optical variability of blazars are given in individual sections.

Markarian galaxies in UV and multiwavelength studies (Mickaelian et al., 2018b). The UV properties of 1152 Markarian galaxies have been investigated based on GALEX data. These objects have been investigated also in other available wavelengths using multi-wavelength data from X-ray to radio. Using our classification for activity types for 779 Markarian galaxies based on SDSS spectroscopy, we have investigated these objects on the GALEX (Bianchi et al., 2011), 2MASS and WISE (Cutri et al., 2013) color-magnitude and color-color diagrams by the location of objects of different activity types and have revealed a number of loci. UV contours overplotted on the optical images revealed additional structures, particularly spiral arms of a number of Markarian galaxies. UV (FUV and NUV) and optical absolute magnitudes and luminosities have been calculated showing graduate transition from AGN to Composites, HIIs and Absorption line galaxies from (average M) $-17.56 \mathrm{~m}$ to $-15.20 \mathrm{~m}$ in FUV, from $-18.07 \mathrm{~m}$ to $-15.71 \mathrm{~m}$ in NUV and from AGN to Composites, Absorption line galaxies and HII from $-21.14 \mathrm{~m}$ to $-19.42 \mathrm{~m}$ in optical wavelengths and from (average L) $7 \times 10^{9}$ to $4 \times 10^{8}$ in FUV, from $1 \times 10^{10}$ to $5 \times 10^{8}$ in NUV and from AGN to Composites, Absorption line galaxies and HII from $7 \times 10^{10}$ to $1 \times 10^{10}$ in optical wavelengths.

Abundance and Star Formation Determinations in Mrk galaxies from SDSS Spectra (Gyulzadyan et al., 2018). We analyze the oxygen and nitrogen abundance and specific star formation rates (sSFR) in Markarian galaxies from Sloan Digital Sky Survey (SDSS) spectra. The Data Release 7 (DR7) of SDSS contains photometric data for more there 1000 and spectral information for more than 700 Markarian objects. The Mrk sample has played a central role in the task of distinguishing between the astrophysical different types of phenomena that occur in AGNs. In the course of the Markarian survey, more than 200 Seyfert galaxies, and hundreds of starbursts, blue compact, and $\mathrm{H}$ II galaxies were discovered. The Markarian survey remains perhaps the best-known source of such objects in the local universe. We have measured their line fluxes and derived the $\mathrm{O}$ and $\mathrm{N}$ abundances using recent calibrations. We have compared the oxygen and nitrogen abundances derived from global emission-line Sloan Digital Sky Survey (SDSS) spectra of galaxies using (1) the Te method and (2) two recent strong-line calibrations: the ON and NS calibrations. The behaviour of the $[\mathrm{N} / \mathrm{H}]$ ratio in under abundant regions gives strong support to a partially primary origin of nitrogen. The star formation rate (SFR) is one of the main parameters used to analyze the evolution of galaxies through time. In the local Universe, the $\mathrm{H} \alpha$ luminosity derived from IFS observations can be used to measure SFR, at least in statistically significant, optically-selected galaxy samples, once stellar continuum absorption and dust attenuation effects are accounted for.

Activity Types of Galaxies Selected from HRC/BHRC Sample (Paronyan et al., 2018, 2019, 2020). In this study we carry out detailed spectral classification of $371(173+198)$ AGN candidates from 
the Joint HRC/BHRC sample, which is a combination of HRC (Hamburg-ROSAT Catalogue, Zickgraf et al. (2003)) and BHRC (Byurakan-Hamburg-ROSAT Catalogue). These objects were revealed as optical counterparts for ROSAT X-ray sources (Voges et al., 1999, 2000); however, spectra for 371 of them are given in SDSS without definite spectral classification. We studied these 371 objects using the SDSS spectra and revealed the detailed activity types for them. Three diagnostic diagrams and direct examination of the spectra were used to obtain more confident classification. We also identified these sources in other wavelength ranges and calculated some of their parameters. In Figure 1 we give examples of SDSS DR15 spectra for some classified HRC-BHRC objects.

Revised optical classification of "LINERs" (Abrahamyan et al., 2018b). This work is dedicated to reclassification of LINERs. For our investigation we use the catalogue Véron-Cetty \& Véron 13th edition (Véron-Cetty \& Véron, 2010). In this catalogue 926 LINERs are included. Cross-correlation of these sources with SDSS DR14 gives 176 objects which have spectra in SDSS. Having mediumresolution spectra from SDSS we have done reclassification of these sources. As a result, $54 \%$ of these sources have changed their classification.

Study and Classification of SDSS Spectra for Byurakan-IRAS Galaxies (Mickaelian et al., 2018a). The sample of Byurakan-IRAS galaxies (BIG) has been created based on optical identifications of IRAS Point Source Catalog (PSC; IRAS (1988)) at high galactic latitudes. As a result, 1178 galaxies have been identified. 172 of them have been observed spectroscopically with Byurakan Astrophysical Observatory (BAO, Armenia) $2.6 \mathrm{~m}$, Special Astrophysical Observatory (SAO, Russia) $6 \mathrm{~m}$ and Observatoire de Haute Provence (OHP, France) $1.93 \mathrm{~m}$ telescopes. Later on, spectra were obtained for more 83 BIG objects in Sloan Digital Sky Survey (SDSS). We have extracted and studied these spectra, classified them and measured spectral features. Diagnostic diagrams have been built to distinguish starbursts (SB), LINERs and Seyfert galaxies. Cross-correlations were made for these objects with multiwavelength (MW) catalogues and their physical properties were studied. Among these 83 objects, 55 HII, 8 Seyfert galaxies, 2 LINERs, 4 other AGN, 6 composite spectrum objects, and 8 other emission-line galaxies have been revealed. Three of these objects are Ultra-Luminous InfraRed Galaxies (ULIRG).

Observations and study of Byurakan-IRAS Galaxies were summarized (Mikayelyan et al., 2019b). A general analysis of optical spectroscopic data on 257 Byurakan-IRAS Galaxies (BIG objects) obtained with the BAO $2.6 \mathrm{~m}$, SAO $6 \mathrm{~m}$, OHP $1.93 \mathrm{~m}$ telescopes and taken from SDSS spectroscopic database was carried out. 149 star-formation regions (SB) galaxies, 42 galaxies with active nuclei (AGN), and 28 galaxies with a composite spectrum were identified. The spectra of 21 galaxies show signs of emission, but without the possibility of more precise determination of their activity type (we assign them as Em), 13 galaxies appear to have star formation rates that do not exceed normal (we assign them as HII), and 3 are absorption galaxies (we assign them as Abs). We give in Table 1 the distribution of 257 BIG objects by activity types.

Table 1. The distribution of 257 BIG objects by activity types.

\begin{tabular}{|c|c|c|c|c|c|}
\hline Activity type & Number of objects & $\mathbf{\%}$ & Activity type & Number of objects & \% \\
\hline HII & 149 & 58.0 & AGN & 13 & 5.1 \\
\hline Composite & 22 & 8.6 & Em & 21 & 8.2 \\
\hline HII / LINER & 4 & 1.5 & Norm & 13 & 5.1 \\
\hline HII / Sy & 2 & 0.8 & Abs & 3 & 1.1 \\
\hline LINER & 12 & 4.6 & Unknown & 1 & 0.4 \\
\hline Sy & 17 & 6.6 & All & $\mathbf{2 5 7}$ & $\mathbf{1 0 0 . 0}$ \\
\hline
\end{tabular}

\section{Discovery of new bright ULIRGs from the IRAS PSC/FSC Combined Catalogue}

(Mikayelyan et al., 2018). High-luminosity IR galaxies (LIRGs, ULIRGs, and HLIRGs) are important for studies related to star-formation processes in the early Universe, as their luminosity allows to detect them at large distances. High IR indicates active star-formation and often starburst processes, which 
is typical to HII (starburst, SB) and AGN. An interesting question is whether the starburst triggers AGN or vice versa or there is no direct impact. Considering that very often such objects manifest double and multiple structure, it is also interesting to investigate the interrelationship between the SB, nuclear activity and interactions or merging. We have analyzed the IRAS PSC/FSC Combined Catalogue for search for new bright ULIRGs. By means of the SDSS DR14 data, namely redshifts for those objects having spectroscopy, we have calculated the IR luminosities and have found 114 very high-luminosity IR galaxies; 107 ULIRGs and 7 HLIRGs. Among them, 48 new ULIRGs and 7 new HLIRGs have been discovered. These objects have been studied by SDSS color-color, luminosityredshift and other diagrams. Further studies will include the content of the sample for activity types and other available data.

Spectral Classification of ULIRGs from IRAS PSC/FSC Combined Catalogue (Mikayelyan et al., 2019a). High-luminosity IR galaxies (LIRGs, ULIRGs, and HLIRGs) are important for studies related to star-formation processes in the early Universe, as their luminosity allows to detect them at large distances. High IR indicates active star-formation and often starburst processes, which is typical to HII (starburst, SB). In many cases high IR indicates an Active Galactic Nuclei (AGN). An interesting question is whether the starburst triggers AGN or vice versa or there is no direct impact. Considering that very often such objects manifest double and multiple structure, it is also interesting to investigate the interrelationship between the SB, nuclear activity and interactions or merging. As a result of cross-correlation of the IRAS PSC/FSC Combined catalogue with SDSS DR14, 114 ULIRGs were separated and classified by the activity types. 1 BLL, 2 quasars, 29 Seyferts of types 1.0-1.8, 5 Seyferts of type 2, 14 LINERS, 36 HII, 14 objects with a composite spectrum (Composite) were identified. Among the type 1 Seyfert galaxies there are many objects with narrow lines.

Radio variable sources at $1400 \mathrm{MHz}$ and their optical variability (Abrahamyan et al., 2018a). In the present study we have cross-correlated NVSS (Condon et al., 1998) and FIRST (Helfand et al., 2015) radio catalogues having radio flux measurements at the same $1.4 \mathrm{GHz}$ frequency. This way we benefit from repeated observations from both catalogues, as they give more accurate positions and fluxes and more important, reveal large differences between the two measured fluxes, thus allowing to establish radio variability. As a result, 79,382 radio variables have been revealed, including 6301 with flux differences at $1.4 \mathrm{GHz}$ larger than $15 \mathrm{mJy}, 1917$ with flux differences $>45 \mathrm{mJy}$ and 260 with flux differences $>200$ mJy. By using a special technique (Mickaelian \& Sinamyan, 2010, Mickaelian et al., 2011), 2425 optically variable objects out of 6301 radio sources have been revealed. 2425 radio sources with both high radio and optical variability into four categories have been divided. 1206 (19\%) out of 6301 radio sources have activity types from available catalogues and 619 (25.5\%) out of 2425 radio sources with at the same time radio and optical variability have activity types from available catalogues. In addition, 279 radio sources out of 2425 have high variability in optical range. We have established their activity types when available. The IR fluxes and colors for the 6301 variable radio sources have been studied. Color-color diagrams show that most of the "unknown" sources are galaxies. The activity types for $110(42 \%)$ out of 260 extremely high variable radio sources also have been retrieved. We give in Table 2 the distribution of the activity types of 6301 radio sources having radio variability.

Classification of BZCAT objects having uncertain types (Abrahamyan et al., 2019a) was aimed at understanding some optical properties of blazars having uncertain types (BZU) in BZCAT Catalogue v5 (Massaro et al., 2015). Cross-correlation with SDSS revealed 43 BZU objects that have spectra in SDSS out of the total 227 BZU ones. We have carried out spectral re-classification for these 43 blazar candidates for activity types. As a result, 37 (86\%) objects out of 43 changed their previous type.

Optical variability of blazars (Abrahamyan et al., 2019b). The analysis of blazars' parameters from BZCAT leads to a conclusion that they do not have the same properties. The preliminary criterion to include an object in the catalog was the strong radio emission; however, two type of radio sources were selected: BL Lacertae (BLL) objects and Flat Spectrum Radio Quasars (FSRQ). As 
Table 2. Activity types of 6301 radio sources having radio variability.

\begin{tabular}{|c|c|c|}
\hline No. & Activity Type & Numbers \\
\hline 1 & Blazar (BZB, BZG, BZQ, BZU) & 308 \\
\hline 2 & QSO & 639 \\
\hline 3 & Sy $1.0 /$ Sy 1 & 19 \\
\hline 4 & Sy 1.2 & 2 \\
\hline 5 & Sy 1.5 & 6 \\
\hline 6 & Sy 1.9 & 2 \\
\hline 7 & Sy 2.0 / Sy 2 & 9 \\
\hline 8 & AGN & 97 \\
\hline 9 & Starburst & 87 \\
\hline 10 & FSS (Flat-Spectrum radio source) & 36 \\
\hline 11 & USS (Ultra-Steep-Spectrum radio source) & $\mathbf{1 2 0 6} \mathbf{( 1 9 \% )}$ \\
\hline & Known (total) & $\mathbf{5 0 9 5} \mathbf{( 8 1 \% )}$ \\
\hline & Unknown & $\mathbf{6 3 0 1} \mathbf{( 1 0 0 \% )}$ \\
\hline & Total & \\
\hline
\end{tabular}

a number of properties are typical of blazars (strong radio emission, optical variability, continuum optical spectra, polarization, high luminosity, etc.), using the optical data, we investigate them to clarify which property plays the most significant role in their classification as blazars. We found that $60 \%$ of blazars have optical variability. We use a technique developed based on POSS1 and POSS2 photometry and group the variability into extreme, strong, medium, and low classes. In the optical range, 51 blazars have powerful variability (extreme variables), and 126 are high variables. In addition, $63 \%$ of blazars have detected radiation in X-ray and $28 \%$ have detected radiation in gamma rays. We give the average statistical characteristics of blazars based on our analysis and calculations.

Fine Analysis of Emission-Line Spectra and Classification of Active Galaxies. Using the SDSS spectroscopy, we have carried out fine optical spectral classification for activity types for 710 AGN candidates. These objects come from a larger sample of some 2500 candidate AGN using preselection by our various samples. More than 800 QSOs have been identified and classified, including 710 QSOs, Seyferts and Composites. The fine classification shows that many QSOs show the same features as Seyferts, i. e. subtypes between S1 and S2 (S1.2, S1.5, S1.8 and S1.9). We have introduced subtypes for the QSOs: QSO1.2, QSO1.5, QSO1.8, QSO1.9, though the last subtype does not appear in SDSS wavelength range due to mostly highly redshifted HVT (the main line for identification of the 1.9 subtype). We also have classified many objects as Composites, spectra having composite characteristics between Sy and LINERs, Sy and HII or LINERs and HII; in some cases all three characteristics appear together resulting as Sy/LINER/HII subtype (Mickaelian et al., 2020). We have used the following works for deriving our fine classification of active galaxies: Heckman (1980), Hoffmeister (1929), Khachikian \& Weedman (1974), Oke \& Gunn (1974), Osterbrock (1981), Osterbrock \& Pogge (1985), Schmidt (1963), Schmitt (1968), Seyfert (1943), Strittmatter et al. (1972), Terlevich (1997, 2000), Thuan \& Martin (1981), Véron et al. (1997), Weedman (1977).

\section{Summary}

A number of interesting results have been obtained in the field of multiwavelength studies of active galaxies, including X-ray, IR and radio sources identified with AGN and Starbursts. Among the important results one could mention:

- Study of SDSS spectra of 371 optical identifications of HRC/BHRC Sample ROSAT X-ray sources (Paronyan et al., 2018, 2019, 2020). These sources were also identified in other wavelength ranges and some of their parameters were calculated. 
- The UV properties of 1152 Markarian galaxies were investigated based on GALEX data (Mickaelian et al., 2018b). Using our classification for activity types for 779 Markarian galaxies based on SDSS spectroscopy, we have investigated these objects on the GALEX, 2MASS and WISE color-magnitude and color-color diagrams by the location of objects of different activity types and have revealed a number of concentrations.

- Abundance and Star Formation Determinations in more than 700 Markarian galaxies from SDSS Spectra (Gyulzadyan et al., 2018). We have measured their line fluxes and derived the $\mathrm{O}$ and $\mathrm{N}$ abundances using recent calibrations. The behavior of the $[\mathrm{N} / \mathrm{H}]$ ratio in under-abundant regions gives strong support to a partially primary origin of nitrogen.

- Revised optical classification of objects classified as "LINERs" in the Catalogue Véron-Cetty \& Véron 13th edition (Abrahamyan et al., 2018b), where 926 LINERs are included. We found 176 objects having spectra in SDSS DR14. The re-classification of these objects led to $54 \%$ of them to change their activity types.

- Study and classification of SDSS spectra for Byurakan-IRAS Galaxies (Mickaelian et al., 2018a). To the previously observed 172 BIG objects (with BAO, SAO and OHP telescopes) 83 BIG objects were added having spectra in SDSS. Among them, 55 HII, 8 Seyfert galaxies, 2 LINERs, 4 other AGN, 6 composite spectrum objects, and 8 other emission-line galaxies have been revealed. Three of these objects are ULIRGs. The observations and study of 257 Byurakan-IRAS Galaxies were summarized in Mikayelyan et al. (2019b).

- Discovery of new bright ULIRGs from the IRAS PSC/FSC Combined Catalogue (Mikayelyan et al., 2018). By means of the SDSS DR14 data, namely redshifts for those objects having spectroscopy, we have calculated the IR luminosities and have found 114 very high-luminosity IR galaxies; 107 ULIRGs and 7 HLIRGs. Among them, 48 new ULIRGs and 7 new HLIRGs have been discovered. Spectral classification of these ULIRGs were carried out (Mikayelyan et al., 2019a). 1 BLL, 2 quasars, 29 Seyferts of types 1.0-1.8 (including narrow-line), 5 Seyferts of type 2, 14 LINERS, 36 HII, 14 objects with a composite spectrum (Composite) were identified.

- Radio variable sources at $1400 \mathrm{MHz}$ were studied and their optical variability waa revealed (Abrahamyan et al., 2018a). As a result, 79,382 radio variables have been revealed, including 6301 with flux differences at $1.4 \mathrm{GHz}$ larger than $15 \mathrm{mJy}$. By using a special technique (Mickaelian \& Sinamyan, 2010, Mickaelian et al., 2011), 2425 optically variable objects out of 6301 radio sources have been revealed.

- Classification of BZCAT objects having uncertain types was carried out (Abrahamyan et al., 2019a). 43 BZU objects were found to have SDSS spectra and as a result, 37 (86\%) objects changed their previous type.

- Optical variability of blazars was studied (Abrahamyan et al., 2019b). We found that $60 \%$ of blazars have optical variability. In the optical range, 51 blazars have powerful variability (extreme variables), and 126 are high variables. In addition, $63 \%$ of blazars have detected radiation in $\mathrm{X}$-ray and $28 \%$ have detected radiation in gamma rays. 
We give in Table 3 a Summary of Projects on Search and Studies of Active Galaxies by our group during 1990s-2010s.

Table 3: Summary of Projects on Search and Studies of Active Galaxies.

\begin{tabular}{|c|c|c|c|c|}
\hline Project & Years & Objectives & Objects & References \\
\hline FBS QSOs/Seyferts & $1986-2001$ & $\begin{array}{l}\text { Bright QSOs } \\
\text { and Seyferts }\end{array}$ & 1,103 & $\begin{array}{l}\text { Mickaelian (2000a, 2003, 2004a, 2008), } \\
\text { Mickaelian et al. (1999, 2001b, 2002a) }\end{array}$ \\
\hline IRAS BIG sample & $1994-2010$ & $\begin{array}{l}\text { AGN, SB, } \\
\text { ULIRGs }\end{array}$ & 1,178 & $\begin{array}{l}\text { Mickaelian (1995, 1997, 2000b, 2001a,b, } \\
\text { 2002), } \\
\text { Mickaelian \& Gigoyan (1998a,b), } \\
\text { Mickaelian \& Sargsyan (2004a,b), } \\
\text { Mickaelian et al. (1997) }\end{array}$ \\
\hline IRAS BIG spectroscopy & $1997-2019$ & $\begin{array}{l}\text { AGN, SB, } \\
\text { ULIRGs }\end{array}$ & 257 & $\begin{array}{l}\text { Mickaelian (2003, 2004b), } \\
\text { Balaian et al. (2001), } \\
\text { Mickaelian \& Sargsyan (2010), } \\
\text { Sargsyan \& Mickaelian (2006), } \\
\text { Mickaelian et al. (1998, 2001a, 2002b, 2003, } \\
\text { 2018a, 2019) }\end{array}$ \\
\hline ROSAT BSC/FSC sources & $2002-2006$ & $\begin{array}{l}\text { Search for } \\
\text { new AGN }\end{array}$ & 6,003 & $\begin{array}{l}\text { Véron-Cetty et al. (2004), } \\
\text { Mickaelian et al. (2006) }\end{array}$ \\
\hline Bright AGN & 2001-pres. & $\begin{array}{l}\text { Statistical } \\
\text { analysis }\end{array}$ & $\sim 10,000$ & \\
\hline Mrk galaxies & 2010-pres. & $\begin{array}{l}\text { Spectral and } \\
\text { MW studies }\end{array}$ & 1,515 & $\begin{array}{l}\text { Mickaelian et al. (2013, 2018b), } \\
\text { Gyulzadyan et al. (2018) }\end{array}$ \\
\hline $\begin{array}{l}\text { IRAS PSC/FSC } \\
\text { extragalactic sample }\end{array}$ & 2011-pres. & $\begin{array}{l}\text { Large IR galax- } \\
\text { ies sample }\end{array}$ & 145,902 & $\begin{array}{l}\text { Hovhannisyan et al. (2011), } \\
\text { Abrahamyan et al. (2015) }\end{array}$ \\
\hline $\begin{array}{l}\text { IRAS PSC/FSC } \\
\text { ULIRGs }\end{array}$ & $2018-2019$ & $\begin{array}{l}\text { IRAS PSC/FSC } \\
\text { ULIRGs }\end{array}$ & 114 & Mikayelyan et al. $(2018,2019 a)$ \\
\hline Spitzer ULIRGs & $2003-2010$ & $\begin{array}{l}\text { High IR/opt flux } \\
\text { ratio galaxies }\end{array}$ & 32 & Sargsyan et al. (2008) \\
\hline SB/AGN Spitzer spectra & 2011 & $\begin{array}{l}\text { SB/AGN Spitzer } \\
\text { spectra }\end{array}$ & 301 & Sargsyan et al. (2011) \\
\hline HRC/BHRC AGN & 2010-pres. & $\begin{array}{l}\text { AGN content in } \\
\text { X-ray }\end{array}$ & 4,253 & $\begin{array}{l}\text { Mickaelian et al. }(2016), \\
\text { Paronyan et al. }(2018,2019,2020)\end{array}$ \\
\hline $\begin{array}{l}\text { NVSS-FIRST variability, } \\
\text { etc. }\end{array}$ & 2013-pres. & $\begin{array}{l}\text { Study of radio } \\
\text { variability }\end{array}$ & 6,301 & Abrahamyan et al. (2018a) \\
\hline Radio AGN & 2020 & $\begin{array}{l}\text { Contents of radio } \\
\text { AGN }\end{array}$ & 1,864 & Abrahamyan et al. (2020) \\
\hline LINERs & 2018 & $\begin{array}{l}\text { Physical proper- } \\
\text { ties of LINERs }\end{array}$ & & Abrahamyan et al. (2018b) \\
\hline ROSAT-NVSS sources & 2013-pres. & $\begin{array}{l}\text { Search for new } \\
\text { AGN, statistics }\end{array}$ & 9,193 & Paronyan et al. (2014) \\
\hline MW study of Blazars & 2014-pres. & $\begin{array}{l}\text { New Blazars, } \\
\text { definition }\end{array}$ & 3,561 & Abrahamyan et al. $(2019 a, b)$ \\
\hline $\begin{array}{l}\text { Fine analysis of emission- } \\
\text { line spectra }\end{array}$ & $2001-2007$ & $\begin{array}{l}\text { Physical proper- } \\
\text { ties of AGN }\end{array}$ & 90 & Kazarian \& Mickaelian (2007) \\
\hline SBS QSOs in WISE & 2017 & QSOs & 450 & Erastova \& Mickaelian (2017) \\
\hline DFBS AGN & 2002-pres. & $\begin{array}{l}\text { New bright } \\
\text { active galaxies }\end{array}$ & $\sim 10,000$ & \\
\hline $\begin{array}{l}\text { Fine classification of active } \\
\text { galaxies }\end{array}$ & 2006-pres. & $\begin{array}{l}\text { Accurate types } \\
\text { and subtypes }\end{array}$ & $\sim 10,000$ & Mickaelian et al. (2020) \\
\hline
\end{tabular}




\section{References}

Abrahamyan H. V., Mickaelian A. M., Knyazyan A. V., 2015, A\&C, 10, 99

Abrahamyan H. V., Mickaelian A. M., Paronyan G. M., Mikayelyan G. A., Gyulzadyan M. V., 2018a, A\&C, 25, 176

Abrahamyan H. V., Mickaelian A. M., Mikayelyan G. A., Paronyan G. M., 2018b, ComBAO, 65, 1

Abrahamyan H. V., Mickaelian A. M., Paronyan G. M., Mikayelyan G. A., Gyulzadyan M. V., 2019a, ComBAO, 66, 1

Abrahamyan H. V., Mickaelian A. M., Paronyan G. M., Mikayelyan G. A., 2019b, AN, 340, 437

Abrahamyan H. V., Mickaelian A. M., Paronyan G. M., Mikayelyan G. A., 2020, Ap, 63, 322

Ahumada R., Allende Prieto C., Almeida A., et al 2020, ApJS, 249, 3

Arakelian M. A., 1975, ComBAO, 47, 3

Baier F. W., Petrosyan M. B., Tiersch H., Shakhbazyan R. K., 1974, Ap, 10, 327

Balaian S. K., Akopian S. A., Mickaelian A. M., Burenkov A. N., 2001, AstL, 27, 284

Bianchi L., Herald J., Efremova B., Girardi L., Zabot A., Marigo P., Conti A., Shiao B., 2011, Ap\&SS, 335, 161

Bird A. J., et al., 2010, ApJS, 186, 1

Condon J. J., Cotton W. D., Greisen E. W., Yin Q. F., Perley R. A., Taylor G. B., Broderick J. J., 1998, AJ, 115, 1693

Cutri R. M., Wright E. L., Conrow T., et al 2013, VizieR Online Data Catalog: AllWISE Data Release, VizieR On-line Data Catalog: II/328. Originally published in: IPAC/Caltech

Erastova L. K., Mickaelian A. M., 2017, AApTr, 30, 261

Gyulzadyan M. V., Mickaelian A. M., Abrahamyan H. H., Mikayelyan G. A., Paronyan G. M., 2018, ComBAO, 65, 392

Heckman T. M., 1980, A\&A, 87, 152

Helfand D. J., White R. L., Becker R. H., 2015, ApJ, 801, 26

Hoffmeister C., 1929, AN, 236, 233

Hovhannisyan A., Sargsyan L. A., Mickaelian A. M., Weedman D. W., 2011, Ap, 54, 147

IRAS 1988, Joint IRAS Science W.G., IRAS Catalog of Point Sources, Version 2.0

Kazarian M. A., Mickaelian A. M., 2007, Ap, 50, 127

Kazarian M. A., Adibekyan V. Z., McLean B., Allen R. J., Petrosian A. R., 2010, Ap, 53, 57

Khachikian E. Y., Weedman D. W., 1974, ApJ, 192, 581

Markarian B. E., Stepanian J. A., Erastova L. K., 1987, IAU S121, p. 25

Markarian B. E., Lipovetsky V. A., Stepanian J. A., Erastova L. K., Shapovalova A. I., 1989, ComSAO, 62, 5

Massaro E., Maselli A., Leto C., Marchegiani P., Perri M., Giommi P., Piranomonte S., 2015, Ap\&SS, 357, 75

Mazzarella J. M., Balzano V. A., 1986, ApJS, 62, 751

Mickaelian A. M., 1995, Ap, 38, 349

Mickaelian A. M., 1997, Ap, 40, 1

Mickaelian A. M., 2000a, AApTr, 18, 557

Mickaelian A. M., 2000b, Ap, 43, 309

Mickaelian A. M., 2001a, Ap, 44, 33

Mickaelian A. M., 2001b, Ap, 44, 185

Mickaelian A. M., 2002, Ap, 45, 288

Mickaelian A., 2003, AApTr, 22, 753

Mickaelian et al. 
Mickaelian A. M., 2004a, BalA, 13, 655

Mickaelian A. M., 2004b, Ap, 47, 361

Mickaelian A. M., 2008, AJ, 136, 946

Mickaelian A. M., 2017, ComBAO, 64, 15

Mickaelian A. M., Gigoyan K. S., 1998a, Ap, 41, 161

Mickaelian A. M., Gigoyan K. S., 1998b, Ap, 41, 232

Mickaelian A. M., Sargsyan L. A., 2004a, Ap, 47, 91

Mickaelian A. M., Sargsyan L. A., 2004b, Ap, 47, 213

Mickaelian A. M., Sargsyan L. A., 2010, Ap, 53, 483

Mickaelian A. M., Sinamyan P. K., 2010, MNRAS, 407, 681

Mickaelian A. M., Gigoyan K. S., Russeil D., 1997, Ap, 40, 379

Mickaelian A. M., Akopian S. A., Balaian S. K., Burenkov A. N., 1998, AstL, 24, 635

Mickaelian A. M., Gonçales A. C., Véron-Cetty M. P., Véron P., 1999, Ap, 42, 1

Mickaelian A. M., Balayan S. K., Hakopian S. A., 2001a, AApTr, 20, 315

Mickaelian A. M., Gonçalves A. C., Véron-Cetty M. P., Véron P., 2001b, Ap, 44, 14

Mickaelian A. M., Abrahamian H. V., Guibert J., Chesnel R., 2002a, Ap, 45, 73

Mickaelian A. M., Hakopian S. A., Balayan S. K., Dodonov S. N., Afanasiev V. L., Burenkov A. N., Moiseev A. V., 2002b, BulSAO, 53, 144

Mickaelian A. M., Hovhannisyan L. R., Sargsyan L. A., 2003, Ap, 46, 177

Mickaelian A. M., Hovhannisyan L. R., Engels D., Hagen H. J., Voges W., 2006, A\&A, 449, 425

Mickaelian A. M., Mikayelyan G. A., Sinamyan P. K., 2011, MNRAS, 415, 1061

Mickaelian A. M., Abrahamyan H. V., Paronyan G. M., Harutyunyan G. S., 2013, AN, 334, 887

Mickaelian A. M., Paronyan G. M., Harutyunyan G. S., Abrahamyan H. V., Gyulzadyan M. V., 2016, AApTr, 29, 333

Mickaelian A. M., Harutyunyan G. S., Sarkissian A., 2018a, AstL, 44, 351

Mickaelian A. M., Abrahamyan H. V., Gyulzadyan M. V., Paronyan G. M., Mikayelyan G. A., 2018b, Ap\&SS, 363, 237

Mickaelian A. M., Abrahamyan H. V., Paronyan G. M., Mikayelyan G. A., Gyulzadyan M. V., 2019, ComBAO, 66, 173

Mickaelian A. M., Abrahamyan H. V., Paronyan G. M., Mikayelyan G. A., 2020, Frontiers in Astronomy and Space Sciences

Mikayelyan G. A., Mickaelian A. M., Abrahamyan H. V., Paronyan G. M., 2018, ComBAO, 65, 13

Mikayelyan G. A., Mickaelian A. M., Abrahamyan H. V., Paronyan G. M., Gyulzadyan M. V., 2019a, Ap, 62, 452

Mikayelyan G. A., Mickaelian A. M., Abrahamyan H. V., Paronyan G. M., Gyulzadyan M. V., 2019b, ComBAO, 66, 31

Moshir M., Copan G., Conrow T., et al 1989, IRAS Faint Source Catalog, Version 2.0, NASA

Oke J. B., Gunn J. E., 1974, ApJL, 189, L5

Osterbrock D. E., 1981, ApJ, 249, 462

Osterbrock D. E., Pogge R. W., 1985, ApJ, 297, 166

Paronyan G. M., Abrahamyan H. V., Harutyunyan G. S., Mickaelian A. M., 2014, in Mickaelian A. M., Sanders D. B., eds, Vol. 304, Multiwavelength AGN Surveys and Studies. pp 164-165, doi:10.1017/S1743921314003652

Paronyan G. M., Mickaelian A. M., Abrahamyan H. V., Mikayelyan G. A., 2018, ComBAO, 65, 412 
Paronyan G. M., Mickaelian A. M., Harutyunyan G. S., Abrahamyan H. V., Mikayelyan G. A., 2019, Ap, 62, 147

Paronyan G. M., Mickaelian A. M., Abrahamyan H. V., Mikayelyan G. A., 2020, Ap, 63, 166

Sargsyan L. A., Mickaelian A. M., 2006, Ap, 49, 19

Sargsyan L., Mickaelian A., Weedman D., Houck J., 2008, ApJ, 683, 114

Sargsyan L., Weedman D., Lebouteiller V., Houck J., Barry D., Hovhannisyan A., Mickaelian A., 2011, ApJ, 730,19

Schmidt M., 1963, Nature, 197, 1040

Schmitt J. L., 1968, Nature, 218, 663

Seyfert C. K., 1943, ApJ, 97, 28

Strittmatter P. A., Serkowski K., Carswell R., Stein W. A., Merrill K. M., Burbidge E. M., 1972, ApJ, 175, L7

Terlevich R., 1997, RMAA, 6, 1

Terlevich R., 2000, In: Advanced Lectures on the Starburst-AGN Connection, Eds. Aretxaga, I.; Mújica, R.; Kunth, D., World Scientific, p. 279

Thuan T. X., Martin G. E., 1981, ApJ, 247, 823

Véron-Cetty M. P., Véron P., 2010, A\&A, 518, A10

Véron-Cetty M. P., et al., 2004, A\&A, 414, 487

Véron P., Gonçales A. C., Véron-Cetty M. P., 1997, A\&A, 319, 52

Voges W., et al., 1999, A\&A, 349, 389

Voges W., Aschenbach B., Boller T., et al 2000, MPE, Garching. The ROSAT Faint Source Catalogue

Weedman D. W., 1977, Vistas in Astronomy, 21, 55

Zickgraf F. J., Engels D., Hagen H. J., Reimers D., Voges W., 2003, A\&A, 406, 535 